\title{
Immunocompetence of children with frequent respiratory infections
}

\author{
LORRAINE J BEARD, GEORGE M MAXWELL, AND Y H THONG \\ Department of Paediatrics, University of Adelaide, Adelaide Children's Hospital, Australia
}

SUMMARY 119 children with recurrent respiratory infections were investigated for immunocompetence. They were divided into two groups. The first, group A, comprised children who had had predominantly upper respiratory infections. Group B comprised those who had had repeated pneumonia in addition. The groups were comparable for gender and age (mean $4 \cdot 2$ years). All the children had reduced neutrophil chemotaxis, reduced neutrophil fungicidal capacity, and perhaps reduced T-cell percentages. Group B children had, in addition to the above, decreased neutrophil bactericidal capacity and a more marked depression of neutrophil chemotaxis. In both groups, some children had reduced immunoglobulin concentrations while others had either normal or increased concentrations. Serum complement, neutrophil iodination, and mitogen-induced lymphocyte transformation were comparable with adult controls in both groups.

Acute respiratory infection constitutes an important problem in paediatrics because of its relative frequency, high morbidity, and occasional mortality. It has been estimated ${ }^{1}$ that each child acquires about 6 respiratory infections a year. There is however, a small group of children in whom respiratory infections are even more common and for which no obvious cause can be found. Few studies have been done to assess the immunocompetence of such children. In 1973, Donovan and Soothill ${ }^{2}$ reported that children with recurrent tonsillitis had lower IgA levels, and suggested ${ }^{2-3}$ that a wider range of tests might help to clarify the problem.

Many children suffer from recurrent upper respiratory infections, especially in the winter. Such children have been assumed to have a deficiency of host defence mechanisms. Treatments aimed at reducing the frequency of such infections have included the use of antibiotics, vitamins, gammaglobulin injections, and more recently, levamisole. ${ }^{4-5}$ Whether or not such children are actually immunodeficient is unknown. In the present studies, we have assessed the immunocompetence of such children with a wide range of in vitro tests, and compared the values with an age-matched group of children with lower respiratory tract infections.

\section{Patients and methods}

We reviewed the case notes of 119 children referred to the Immunology Service during the last 3 years for predominantly respiratory complaints. Two categories of children could be distinguished in this sample. The first (group A) comprised children who had had repeated upper respiratory tract infections, defined as 6 or more a year for older children, or more than 1 over 2 months for infants; such infections included rhinitis, otitis media, tonsillitis, sinusitis, laryngotracheobronchitis. Group B comprised children who, in addition to having upper respiratory infections, also had had at least 2 episodes of pneumonia, or, in the case of infants, pneumonia of unusual severity. Group A comprised 45 boys and 36 girls; their ages ranged from 0.5 to 15.0 years, with a mean $( \pm S D)$ of $4 \cdot 3 \pm 3.4$. Group B comprised 23 boys and 15 girls; their ages ranged from 0.2 to 15.1 years, with a mean of $4 \cdot 1 \pm 3 \cdot 1$. Obvious causes of their respiratory infections-such as cystic fibrosis, foreign body in the respiratory tract, or gastro-oesophageal refluxwere excluded by appropriate clinical and radiological assessment and laboratory testing. Children with recognisable immunodeficiency syndromes ${ }^{6}$ such as selective IgA deficiency (4 cases), myeloperoxidase deficiency, ${ }^{7}$ or Shwachman's syndrome ${ }^{8}$ were excluded from this analysis.

Immunological tests were performed when the children were relatively well and free from infection. The serum immunoglobulins (IgA, IgG, IgM) and complement $(\mathrm{C} 3, \mathrm{C} 4)$ were measured by radial 
immunodiffusion using commercial plates (Behringwerke, W. Germany), total haemolytic complement by lysis of sheep red cells, ${ }^{9}$ lymphocytes and neutrophils were purified from heparinised blood by a one-step centrifugation procedure, ${ }^{10} \mathrm{~T}$-cells were counted by spontaneous rosetting with sheep red cells, ${ }^{11}$ lymphocyte response to mitogens was measured by a microculture technique, ${ }^{12}$ neutrophil iodination by a microassay procedure ${ }^{13}$ bactericidal activity by reduction of Staphylococcus aureus colony counts, ${ }^{14}$ fungicidal capacity by reduction of Torulopsis glabrata colony counts, ${ }^{15}$ and chemotaxis by neutrophil migration under agarose. ${ }^{16}$ All these tests could generally be done, using microtechniques, on 5 to $7 \mathrm{ml}$ blood. However, in a few instances some tests were omitted because of inadequate blood sampling. There were 175 healthy adult controls. The results were analysed by Student's $t$ test for unpaired data.

\section{Results}

The serum immunoglobulin concentrations of each patient are presented in Figs 1-3. The normal values for children were obtained from two reports. ${ }^{17-18}$ The results show that 7 children in group $A$ and 3 children in group B had low levels of IgG (Fig. 1) while increased IgG was found in 5 from group $A$ and 7 from group $B$. There were 9 children from group A and 3 from group B with low IgA levels (Fig. 2) while 3 from group A and 1 from group B had high IgA. There were 2 from each group with low IgM (Fig. 3) and 11 from group A and 9 from group B with increased IgM.

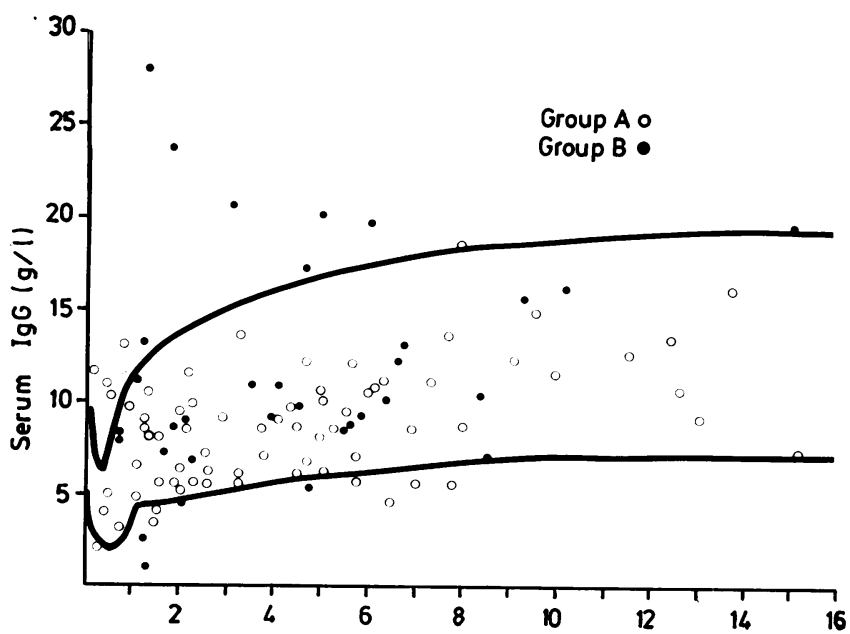

Age (years)

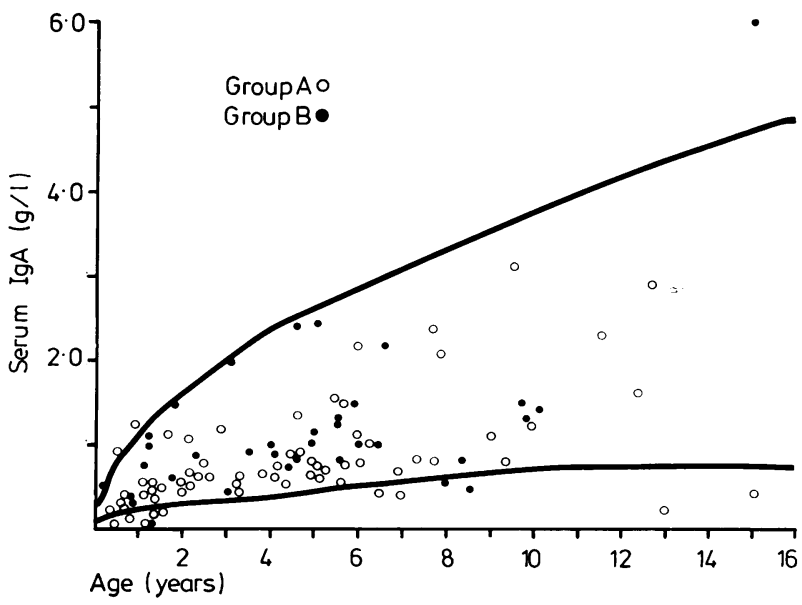

Fig. 1 Serum IgG concentrations in children with repeated respiratory infection: group $A$ upper respiratory tract, group B pneumonia. Results are plotted on graph depicting $2 S D^{17-18} \operatorname{Ig} G$ concentrations in relation to age.

Fig. 2 Serum IgA concentrations in children with repeated respiratory infection. Results are plotted on graph depicting $2 S D^{17-18} \operatorname{Ig} A$ concentrations in relation to age. 


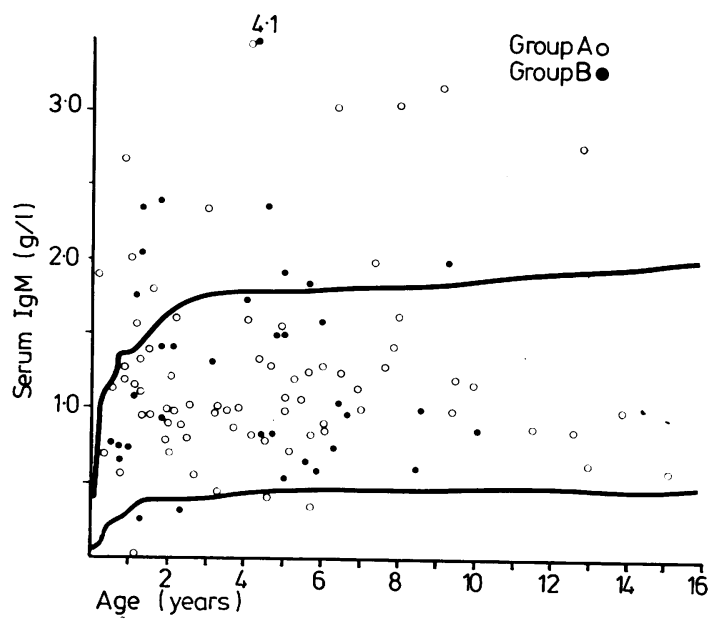

Fig. 3 Serum IgM concentrations in children with repeated respiratory infection. Results are plotted on graph depicting $2 S D^{17-18}$ IgM concentrations in relation to age.

Total haemolytic complement values for group A were $133 \pm 32$ (mean $\mathrm{U} / \mathrm{ml} \pm \mathrm{SD}$ ) compared with $127 \pm 35$ in group $B$. The difference was not statistically significant $(P>0.5)$, and both groups were within the range of normal for our laboratory $(95-165 \mathrm{U} / \mathrm{ml})$. Similar results were obtained for C3 and C4. For C3, group A showed $1.02 \pm 0.24$ compared with $1 \cdot 10 \pm 0 \cdot 32 \mathrm{~g} / 1$ in group $\mathrm{B}$, both being within the normal range of $0 \cdot 65-1 \cdot 60$. For $\mathrm{C} 4$, group A showed $0.36 \pm 0.12$ compared with $0.34 \pm 0.10 \mathrm{~g} / \mathrm{l}$ in group $\mathrm{B}$, both being within the normal range of $0 \cdot 20-0 \cdot 40$.

T-cell percentages in both groups were slightly lower than in adult controls (Fig. 4) being $53.3 \pm 11.0$ (mean \pm SD) in group $A$ and $54.9 \pm 10.0$ in group B, compared with $59.4 \pm 7 \cdot 4$ in controls $(P<0 \cdot 01)$. However, no significant difference was found between the groups $(P>0 \cdot 4)$.

The resting ${ }^{3} \mathrm{H}$-thymidine uptake of lymphocyte cultures was found to be appreciably higher in group B than in group A and adult controls (Fig. 4). Mean counts per minute \pm SD for group $B$ were $1118 \pm 987$ compared with $760 \pm 669$ for group $A$ $(P<0.02)$ and $841 \pm 802$ in controls $(P<0.02)$.

There was no significant difference between either group and controls in their responses to phytohaemagglutinin and pokeweed mitogen. However, lymphocyte response to concanavalin $\mathrm{A}$ was significantly raised in group B compared with group A or controls (Fig. 4). Counts per minute (mean \pm SD) were $31490 \pm 18759$ in group $B$ compared with $22410 \pm 11908$ in group $A(P<0 \cdot 01)$,
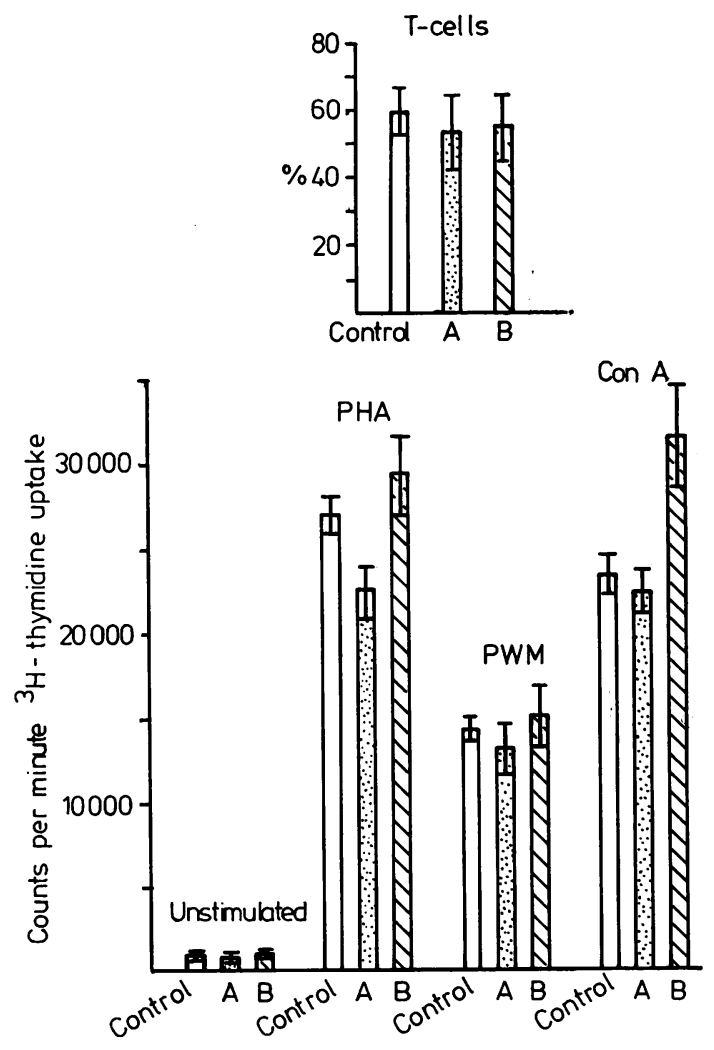

Fig. 4 T-cell percentages and lymphocyte mitogenic responses of children with repeated respiratory infections. Results for T-cells are shown as percentage mean $\pm S D$. Results for lymphocyte mitogenic responses are shown as counts per minute (mean $\pm S E$ ) ${ }^{3} \mathrm{H}$-thymidine uptake.

and $23373 \pm 13421$ in controls $(P<0.01)$. There was no significant difference between group $\mathrm{A}$ and controls $(P>0 \cdot 5)$.

Neutrophil chemotaxis was significantly decreased in both groups (Fig. 5). In group A it was $1 \cdot 20 \pm 0 \cdot 51$ (mean $\mathrm{mm} / 3$ hours $\pm \mathrm{SD}$ ) compared with $1.58 \pm$ 0.41 in controls $(P<0.001)$. In group $B$ it was even less than in group $A$, with a value of $0.81 \pm 0.53$ $(P<0.001)$. In contrast, neutrophil iodination in both groups was similar to control values (Fig. 5). Fungicidal capacity however, was decreased in both groups. Percentage (mean \pm SD) colony reduction was $83 \cdot 6 \pm 9 \cdot 3$ and $81 \cdot 5 \pm 10 \cdot 4$ for groups $A$ and $B$ respectively, compared with $88.0 \pm 5 \cdot 6$ in controls $(P<0.01)$. Only group $B$ had a decrease in bactericidal capacity. Percentage colony reduction in group B was $72 \cdot 6 \pm 20 \cdot 0$ compared with $87 \cdot 4 \pm$ 


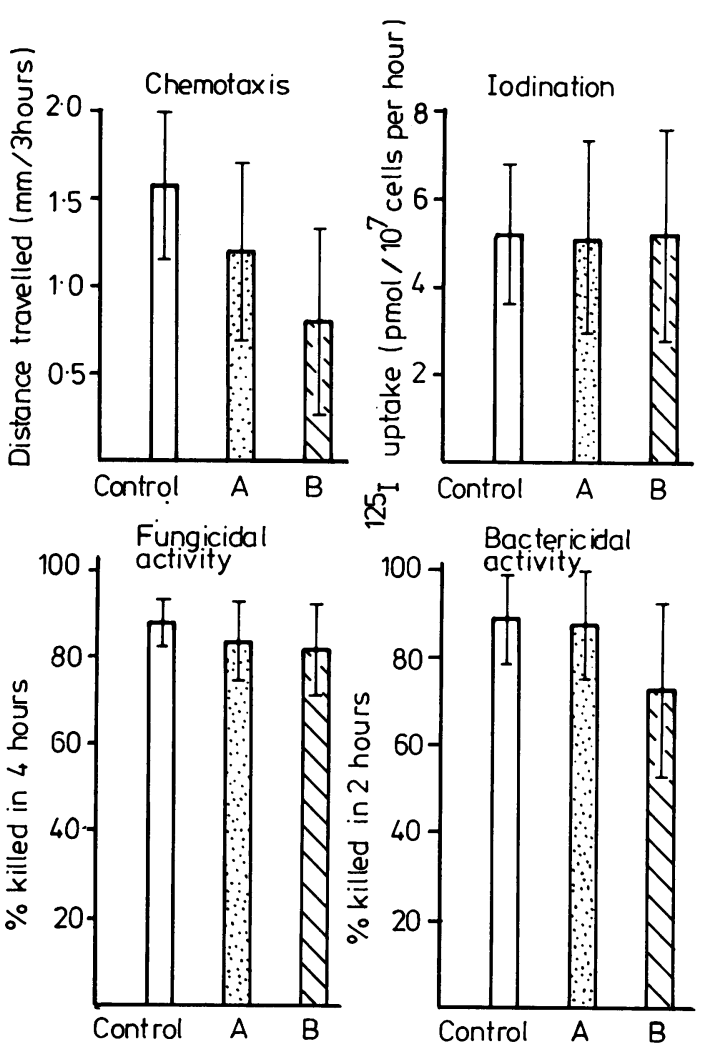

Fig. 5 Neutrophil function of children with repeated respiratory infections. Chemotaxis is expressed as distance travelled in $\mathrm{mm} / 3$ hours (mean $\pm S D$ ). Quantitative iodination is expressed as pmol $/ 10^{7} \mathrm{cells} / \mathrm{h}$ (mean $\pm S D$ ) of ${ }^{125}$ I-iodide incorporated. Fungicidal capacity is expressed as percentage reduction of colonies (mean $\pm S D)$ in 4 hours, and bactericidal capacity as percentage reduction of colonies (mean $\pm S D$ ) in 2 hours.

12.4 in group $A(P<0.001)$ and $88.4 \pm 10.6$ in controls $(\mathrm{P}<0 \cdot 001)$.

\section{Discussion}

The immune capacity of all individuals can be considered to fall on a continuum extending from gross immunodeficiency to excellent immunocompetence. Soothill ${ }^{3}$ has pointed out that the use of the less than 2 SD designation to diagnose immunodeficiency may not give a true picture of clinical events, since individuals on the lower end of the continuum, but not less than 2 SD, who may have a greater susceptibility to infection, would be regarded as having comparable immunocompetence with those at the higher end of this continuum.

This situation can be rectified by statistical analysis of larger patient populations. In the present studies, we have analysed the results of 2 groups of children with repeated respiratory infections, group A with repeated infections generally confined to the upper respiratory tract, and group B with infections affecting the lower respiratory tract (pneumonia). In the less age-related tests-such as complement activity, lymphocyte response, and neutrophil function-the results of the two groups had to be compared with adult controls because of ethical constraints on the recruitment of age-matched children. Such data must be interpreted with care, but additional safeguards are provided by comparing the two groups who, with regard to age and gender, are comparable with each other.

However, because of the age-dependent nature of serum immunoglobulins, the less than 2 SD designation has to be used for analysis. Such analysis is likely to provide an underestimate of the actual number of immunodeficient individuals. Even then, a substantial number of children in both groups was found to have immunoglobulin deficiency.

Children with complement deficiency do not generally present with repeated respiratory infections. ${ }^{19-20} \mathrm{It}$ is therefore not surprising that total haemolytic complement activity, $\mathbf{C} 3$ and $\mathbf{C} 4$ concentrations for both groups of children fell within the normal adult range.

T-cell percentages of both groups were significantly lower than adult controls. Since the values of group A and group B were not statistically different from each other, this difference could be a function of age, rather than an indication of immunodeficiency. The mitogen responses to phytohaemagglutinin and pokeweed mitogen of both groups of children were similar to adult controls and consistent with the general impression that these results are not agedependent, except in the newborn and elderly. ${ }^{21}$ In contrast, both the resting values of ${ }^{3} \mathrm{H}$-thymidine uptake and the response to concanavalin $\mathrm{A}$ in group B were significantly increased, compared with group $A$ and adult controls. The findings are difficult to interpret. Perhaps group B patients, with their more serious lower respiratory tract infections, were responding by increasing the metabolic activity of their lymphocytes.

The iodination capacity in both groups was comparable with adult controls. This test measures both phagocytic and metabolic activity of neutrophils and provides a general assessment of their function. However, the actual fungicidal activity of neutrophils was diminished in both groups of patients, while bactericidal capacity was diminished only in group B. These results suggest that group B children have more impairment of neutrophil function than those in group A. This conclusion is 
further supported by measurements of neutrophil chemotaxis, which also showed that the neutrophils of group B children have a lower chemotactic response than those of group A children, and well below that of controls.

In summary, the results of these studies show that children with repeated respiratory infections mainly affecting the upper respiratory tract had reduced neutrophil chemotaxis and fungicidal capacity, and perhaps reduced T-cell percentages. Those children with pneumonia had, in addition to the above, decreased neutrophil bactericidal capacity and an even greater depression of neutrophil chemotaxis. In both groups, some children had reduced immunoglobulin levels while others had normal or increased levels. The higher-than-normal levels of serum immunoglobulin found in some of these children may be a reactive phenomenon in response to the repeated infections, because of a defect in some other component of the immune system.

The respiratory system is vulnerable to attack by micro-organisms because of the large epithelial surface area in contact with the external environment. The local mechanisms of host defence in the respiratory tract are not well understood, but systemic immune mechanisms are also operative and patients with immunodeficiency syndromes have frequent respiratory infections. ${ }^{6}$ Since we have excluded children with known and well-established immunodeficiency syndromes from the present studies the impairment of immune responses found in the children we studied is all the more relevant. In most instances, the impairment was relatively minor when considered in isolation and assessed by the conventional less than 2 SD criterion. When considered as a group however, statistical analysis showed significant impairment of immune function, more so in the group with lower respiratory tract involvement than the group with predominantly upper respiratory symptoms. These findings lend further support to previous observations ${ }^{2}$ that children with recurrent tonsillitis have reduced immunological responses. They also support Soothill $^{3}$ in that immunodeficiency disorders in children are more common than had generally been thought. Finally, these studies may help to identify and delineate less severe immunodeficiency disorders, one of the recommendations of the World Health Organisation. ${ }^{6}$

\section{References}

1 Hope-Simpson R E, Higgins P G. A respiratory virus study in Great Britain: review and evaluation. Prog Med Virol 1969; 11: 354-407.

2 Donovan R, Soothill J F. Immunological studies in children undergoing tonsillectomy. Clin Exp Immunol 1973; 14: 347-57.
${ }^{3}$ Soothill J F. Immunity deficiency states. In: Gell P D H, Coombs R R A, Lachman P J, eds. Clinical aspects of immunology. Oxford: Blackwell Scientific, 1975: 649-87.

4 Van Eygen M, Znamensky P Y, Heck E, Raymaekers J. Levamisole in prevention of recurrent upper-respiratory tract infections in children. Lancet 1976; i: 382-5.

5 Van Eygen M, Dils F, Gillerot J, Verschueren E. A doubleblind pediatric evaluation of levamisole in the prevention of recurrent upper respiratory tract infections. Eur J Pediatr 1979; 131 : 147-53.

6 World Health Organisation Scientific Group on Immunodeficiency. WHO Report: Immunodeficiency. Clin Immunol Immunopathol 1979; 13: 296-359.

7 Robertson C F, Thong Y H, Hodge G L, Cheney K. Primary myeloperoxidase deficiency associated with impaired neutrophil margination and chemotaxis. Acta Paediatr Scand 1979; 68: 915-9.

8 Thong Y H. Impaired neutrophil kinesis in a patient with the Shwachman-Diamond syndrome. Aust Paediatr J 1978; 14: 34-7.

- Mayer M M. Complement and complement fixation. In: Kabat E A, ed. Experimental immunochemistry, second edition. Springfield, Illinois: Thomas, 1971: 133.

10 Ferrante A, Thong Y H. A rapid one-step procedure for purification of mononuclear and polymorphonuclear leukocytes from human blood using a modification of the Hypaque Ficoll technique. J Immunol Methods 1978; 24: 389-93.

11 Scheinberg M, Blacklow N R, Goldstein A L, Parrino J A, Rose F B, Cathcart E S. Influenza: response of T-cell lymphopenia to thymosin. N Engl J Med 1976; 294: 1208-11.

12 Thong Y H, Ferrante A. Inhibition of mitogen-induced human lymphocyte proliferative responses by tetracycline analogues. Clin Exp Immunol 1979; 35: 443-6.

13 Thong Y H, Ferrante A. A semi-automated microassay technique for quantitative leukocyte iodination. J Immunol Methods 1978; 20: 297-9.

14 Thong Y H, Ferrante A, Ness D. Neutrophil phagocytic and bactericidal dysfunction induced by bilirubin. Aust Paediatr J 1977; 13: 287-9.

15 Thong Y H, Ferrante A. Phagocytosis and killing of Torulopsis glabrata by human neutrophils. IRCS J Med Sci 1977; 5: 482-3.

16 Ferrante A, Beard L J, Thong Y H. Early decay of human neutrophil chemotactic responsiveness following isolation from peripheral blood. Clin Exp Immunol 1980; 39: 532-7.

17 Harpey J P. Immune deficiencies in the infant. Notes on Diagnosis, No 2. Australia: Hoechst, 1977.

18 Shelton M J, Meek F, Geller I, Hosking C S. Serum immunoglobulin levels in children. Aust J Med Technol $1974 ; 5$ : 113-7.

19 Johnston R B, Jr, Stroud R M. Complement and host defense against infection. $J$ Pediatr 1977; 90: 169-79.

20 Thong $\mathrm{Y} \mathbf{H}$, Simpson $\mathrm{D}$ A, Müller-Eberhard $\mathrm{H} \mathrm{J}$. Homozygous deficiency of the second component of complement presenting with recurrent bacterial meningitis. Arch Dis Child 1980; 55: 471-3.

21 Lange C F. Immunology of aging. Prog Clin Pathol 1978; 7: $119-36$.

Correspondence to Dr Y H Thong, Department of Paediatrics, Adelaide Children's Hospital, North Adelaide, South Australia 5006.

Received 26 November 1979 